\title{
Wound healing biomolecules present in four proposed soft aqueous extractions of Ageratum conyzoides Linn
}

\author{
Amivi Edefia AKPALO ${ }^{1,4 *}$, Issa Kouassi SALOUFOU ${ }^{4}$, Kodjo ELOH ${ }^{3}$ and \\ Kafui KPEGBA 2,4 \\ ${ }^{1}$ University of Lome, Department of Biochemistry, BP: 1515 Lome, TOGO. \\ ${ }^{2}$ University of Lome, Department of Chemistry, BP: 404 Lome, TOGO. \\ ${ }^{3}$ University of Kara, Department of Chemistry, BP: 404 Kara, TOGO. \\ ${ }^{4}$ Chemistry and Natural Substances Laboratory (LabCOSNat), BP: 1515 Lome, TOGO. \\ *Corresponding author; E-mail: edefia@gmail.com
}

\begin{abstract}
Biochemically wound healing process is promoted by active agents which compose aqueous and alcohol herbal extracts and those components act synergistically. We propose in this study four methods of soft aqueous extraction (A, B, C, D) of Ageratum conyzoides Linn. leaves, a well-known west african plant for its wound healing properties. Our main objective was to know which of the extraction methods would allow us to obtain specifically tannins and flavonoids. We also investigated which types of flavonoids extracted could inhibit serine proteases like thrombin or serine proteases present in bacterial and then being bactericide. Ageratum conyzoides Linn. samples were collected in the beginning of the raining period in May 2019 in two regions of Togo. After aqueous extractions, TLC and HPLC-ESI+-QTOF analysis were done. Chromatographic fingerprints obtained were quite similar for the four extracts investigated. However, additional spots with different colors appeared for the B extract. Flavonoids were revealed by the numerous colors of spots observed on the chromatograms. Superposing HPLC-ESI ${ }^{+}$-QTOF chromatographic profiles of the four extracts, we deduced that our proposed extraction methods lead to same chemical compounds but B extract contained higher concentrations of later substances. Identified molecules belong to terpernoids and flavonoids classes.

(C) 2020 International Formulae Group. All rights reserved.
\end{abstract}

Keywords: Ageratum Conyzoides Linn., Aqueous extractions, Biomolecules, Wound healing.

\section{INTRODUCTION}

The process of wound healing is promoted by several herbal extracts, which are composed of active agents like triterpenes, alkaloids, flavonoids, tannins, saponins, anthraquinones, and other biomolecules (Akpalo et al., 2015). Tannins act as free radical scavengers. Triterpenoids and flavonoids promote wound healing due to their astringent and antimicrobial properties. Saponins, due to their antioxidant and antimicrobial activity appear to be responsible for wound contraction and elevated rate of epithelialization. More, we know that flavonoids also possess potent antioxidant and free radical-scavenging effect enhancing the rate of antioxidant enzymes in granuloma tissue. The antioxidant and anti-inflammatory activities of flavonoids were believed to be one of the important mechanisms in wound healing and in the presence of tannin, it improved the regeneration and organization of the new tissue 
and hastened the wound healing process. Indeed, biochemically wound healing process is promoted by active agents which compose aqueous and or alcohol herbal extracts and those components act synergistically (Akpalo et al., 2015).

The antioxidant and anti-inflammatory activities of flavonoids were believed to be one of the important mechanisms in wound healing and in the presence of tannin, it improved the regeneration and organization of the new tissue and hastened the wound healing process. Indeed, biochemically wound healing process is promoted by active agents which compose aqueous and or alcohol herbal extracts and those components act synergistically (Akpalo et al., 2015).

The antioxidant and anti-inflammatory activities of flavonoids were believed to be one of the important mechanisms in wound healing and in the presence of tannin, it improved the regeneration and organization of the new tissue and hastened the wound healing process. Indeed, biochemically wound healing process is promoted by active agents which compose aqueous and or alcohol herbal extracts and those components act synergistically (Akpalo et al., 2015).

The increased production of reactive oxygen species during injury results in consumption and depletion of the endogenous scavenging compounds. Flavonoids may have an additive effect to the endogenous scavenging compounds (Panche et al., 2016).

Microbiome analysis of chronic wounds has progressed significantly in recent years. Findings support the hypotheses that microbes contribute to impaired wound healing, and wound microbiomes encompass diverse species of bacteria and fungi are under-appreciated agents of complication (Kalan and Grice, 2018). Flavonoids have also been recognized for their antimicrobial activity and many researchers have isolated and identified the structures of flavonoids having properties of antifungal, antiviral and antibacterial activity (Panche et al., 2016).

Ageratum conyzoides Linn. (Asteraceae), a well-known west african plant for its wound healing properties, is an annual herb with a long history of traditional medicinal used in the tropical and sub-tropical region of the world (Singh et al., 2013). The mature plant is used for its haemostatic, and anti- inflammatory, properties for the treatment of wounds in bacterial infections (Singh et al., 2013). However, Ageratum conyzoides Linn. aqueous leaves extracts prevent coagulation in the whole blood (Singh et al., 2013). It is true thrombin plays a pivotal role in thrombogenesis (Liu et al., 2010). Indeed, in the last decade knowledge, flavonoids were found to belong to a class of serine proteases inhibitors and thrombin is a serine protease.

Then, in this study, we proposed four methods of soft aqueous extraction of leaves of Ageratum conyzoides Linn. Bearing in mind the above considerations, our principal objective was to search to know which of the four extractions methods allows us to obtain specifically tannins and flavonoids. More, we investigated which types of flavonoids we could extract to understand how those extracts could reversibly or irreversibly inhibit serine proteases like thrombin, a key enzyme in hemostasis, the first stage of epidermal wound healing process. Those flavonoids could also inhibit serine proteases present in bacterial, inhibiting protein synthesis and then being bactericide.

\section{MATERIALS AND METHODS Plant material}

Ageratum conyzoides Linn. samples were collected in the beginning of the raining period in May 2019 in two regions: Guerinkouka and Tove. Guerinkouka (GPS coordinates: $9.6896200 ; 0.6079700)$ is situated in the Kara region in Northern Togo and Tove (GPS coordinates: 6.884119; 0.646683) is located in the Plateaus region in Southern Togo. Botanical material was authenticated by the Department of Botanic, University of Lome as “E 9 41'22.6”"N 0³6'28.7”. The fresh material was oven-dried at a temperature not exceeded $37^{\circ} \mathrm{C}$ for a week.

\section{Preparation of extracts}

The method, although greatly modified, was inspired from (Li et al., 2011). Four different derived extracted methods were proposed. The leaves of the plant finely cut (25g in weight) were macerated 15 minutes at RT $\left(25{ }^{\circ} \mathrm{C} \pm 2{ }^{\circ} \mathrm{C}\right)$ and then extracted with distilled water $(250 \mathrm{ml}): 1 \mathrm{~h}$ at $50^{\circ} \mathrm{C} \pm 2{ }^{\circ} \mathrm{C}(\mathrm{A})$, $3 \mathrm{~h}$ at $50{ }^{\circ} \mathrm{C} \pm 2{ }^{\circ} \mathrm{C}(\mathrm{B}), 1 \mathrm{~h}$ at $25^{\circ} \mathrm{C} \pm 2{ }^{\circ} \mathrm{C}$ (C) or $3 \mathrm{~h}$ at $25^{\circ} \mathrm{C} \pm 2{ }^{\circ} \mathrm{C}$ (D). For each method, the plant leaves were extracted three times and 
filtrates were combined, placed in a water bath to dry at $50{ }^{\circ} \mathrm{C} \pm 2{ }^{\circ} \mathrm{C}$. Thereafter, the extracts were weighted and a certain amount of $95 \%$ ethanol was added. The final concentration of extracts solution was obtained by dilution to $80 \%$. This extract solution was deposited overnight at RT $\left(25^{\circ} \mathrm{C} \pm 2{ }^{\circ} \mathrm{C}\right)$ and centrifuged at $4000 \mathrm{rpm}$ for $10 \mathrm{~min}$. The mixture was filtered using a filter paper (Whattman $\mathrm{N}^{\circ} 1$ ) and a part (in ethanol) was used for the study (A, B, C, D). The filtrate was placed in a water bath to dry at $50{ }^{\circ} \mathrm{C} \pm 2{ }^{\circ} \mathrm{C}$ and the clear residue was dissolved this time in a sodium saline solution (A', B', C', D') and used for biochemical and microbiological studies.

\section{Thin layer chromatographic analysis procedure Separation}

The thin layer chromatographic analyzes were carried out in normal phase on aluminum plates coated with a Kieselgel 60 F254 type silica gel. (Merck) $0.25 \mathrm{~mm}$ thick (Saloufou et al., 2017). The eluent is poured to a height of about $0.5 \mathrm{~cm}$ in an elution vessel hermetically sealed to saturation with steam. The samples were deposited on the TLC plate using a glass capillary pipette and the produced stain was dried rapidly between each application. The plate was introduced and placed vertically in the tank which was immediately closed. When the solvent front came about $1 \mathrm{~cm}$ from the top end of the plate where a fine line is previously marked, the plate is removed from the tank. Different eluent systems were tested in order to obtain a better separation of the extracts. The observation of TLC is first carried out under UV light at $254 \mathrm{~nm}$ by fluorescence quenching then at $366 \mathrm{~nm}$ and then revealed by vanillin sulfuric acid or chloride aluminum spray followed by heating and/or UV light exposition. The developers used for this work were prepared as follows:

\section{Vanillin sulfuric acid Developer}

The vanillin sulfuric acid reagent was prepared by dissolving $1 \mathrm{~g}$ of vanillin added with $2 \mathrm{ml}$ of sulfuric acid in $100 \mathrm{ml}$ of $95 \%$ ethanol. After spraying, the TLC plate was heated at $110{ }^{\circ} \mathrm{C}$ for about 5 minutes. Several colorations appear depending on the types of compounds.

\section{Aluminum chloride developer}

The aluminum chloride developer was obtained by dissolving $0.5 \mathrm{~g}$ of the aluminum chloride in $100 \mathrm{ml}$ of ethanol.

\section{HPLC-ESI ${ }^{+}$-QTOF analysis}

A methanolic solution $(1000 \mu \mathrm{g} / \mathrm{mL})$ was prepared from the different ethanolic extracts. The chromatographic analysis for the separation of the compounds was carried out in reverse phase on an Agilent series 1200 HPLC system equipped with a C18 column from Microchip Technology (Agilent, Zorbax 300 SB-C18, $5 \mu \mathrm{m}, 43 \mathrm{~mm}, 75 \mu \mathrm{m})$. An Agilent 6530 quadrupole - time of flight (QTOF) mass detector (Agilent Technologies, Santa Clara, Calif., USA) coupled to an electrospray ionizer (ESI) was used. Chromatographic conditions were as follows: flow rate: $0.4 \mu \mathrm{L} \cdot \mathrm{min}^{-1}$; solvent A: $0.1 \%$ formic acid in water; solvent B: $0.1 \%$ formic acid in methanol. The solvent gradient $(\mathrm{v} / \mathrm{v})$ was generated starting from $20 \%$ $\mathrm{B}$ and reaching $100 \% \mathrm{~B}$ in 10 minutes and maintaining at $100 \% \mathrm{~B}$ for 10 minutes for a total run of $20 \mathrm{~min}$. Two microliters of the samples were analyzed by ESI in positive mode. Mass spectral data were acquired in the range $\mathrm{m} / \mathrm{z} 100-1500$, with an acquisition rate of 1.35 spectra. $\mathrm{s}^{-1}$, averaging 10000 transients. The source parameters were adjusted as follows: drying gas temperature $250{ }^{\circ} \mathrm{C}$, drying gas flow rate $5 \mathrm{~L} \cdot \mathrm{min}^{-1}$, nebulizer pressure 45 psi and voltage fragmentor $150 \mathrm{~V}$. Data acquisitions and processing were done by Agilent Mass Hunter Workstation Acquisition software v. B.02.00. The METLIN database ( https://metlin.scripps.edu/index.php) was used to predict plausible structures of detected metabolites (Saloufou et al., 2018).

\section{RESULTS}

\section{Thin layer chromatography analyses}

The separation was tested many times with different systems of migration solvents. Developing solvent is usually considered as the most important parameter affecting the selectivity in TLC (Vovk et al., 2005). Since the migration solvent Ethylacetate/Dichloromethane (v/v: 30/70) produced the best separation, it was also more informative for the four types of extraction. Only two visualizing agents were tested, vanillin-sulfuric and chloride aluminum. Vanillin-sulfuric allows a vast number of chromatographic bands to appear. Chloride aluminum revealed specifically flavonoids under UV light at $366 \mathrm{~nm}$. 


\section{Phytochemical screening of the four proposed extracts}

Chromatographic fingerprints obtained in visible with Vanillin sulfuric or under UV at $366 \mathrm{~nm}$ with Chloride Aluminium were quite similar for the four extracts investigated Figure 1 and Table 1.

Brown, green, yellow, blue, purple staining were observed in visible with Vanillin Sulfuric in A, C and D extracts. Pink and orange were additionally observed in B extract. With Aluminum Chloride, essentially yellowgreen fluorescence was observed independently of the mode of extraction and the distance of migration. A supplementary band at $\mathrm{Rf}=0.28$ and $\mathrm{Rf}=0.30$ respectively in $\mathrm{C}$ and $\mathrm{D}$ extracts appeared. More, for the D extract, pink staining was mixed with yellow-green. For all the extracts except $\mathrm{B}$, the bands presented between the application point $\mathrm{Rf}=0.00$ and the band at $\mathrm{Rf}=0.88$ were almost fully analogous and they could be considered as characteristic of the modes of extraction. Pale orange at $\mathrm{Rf}$ value 0.29 ; orange additionally to yellow at $\mathrm{Rf}$ value 0.36 , pink instead of blue at Rf value 0.66 and blue color appearing at $\mathrm{Rf}$ value 0.71 were specific to B extract.

Phytochemical screening of extracts A, $\mathrm{B}, \mathrm{C}$ and $\mathrm{D}$ revealed the presence of flavonoids by the numerous colors of spots observed on the chromatograms.

\section{Stability and / or evolution of B extracts solubilized in sodium saline solution}

For this part, we repeated the B extracted method three times again at a week interval and we compared the extracts solubilized in sodium saline solution, a plasma isotonic solution used in hospitals to clean wounds. As the use of the Vanillin Sulfuric visualizing reagent produced more stable results (i.e. the band colors did not considerably change with time) (Cieśla et al., 2011), we did not use chloride aluminium any more for this TLC.

Before all, we noted number and colors of spots differed from those obtained previously when extracts were solubilized in ethanol. Thus, new molecules, especially tannins because of red color, which are water soluble were revealed (Figure 2 and Table 2). More precisely, they are anthocyanidols which react with Vanillin Sulfuric to produce red color.
To go deeper, we observed variations in spots colors intensities (Figure 2 and Table 3). Since TLC were not done just after extractions (see dates Figure 2 and Table 2) but one month later, September $21^{\text {st }}$. Intensities became pale with time. At $\mathrm{Rf}$ values $0.40 / 0.41 / 0.42$ and $\mathrm{Rf}$ values 0.68 , red or orange-red colors intensities progressively changed in pink or pink-orange respectively and finally completely disappeared. We strongly supposed these variations corresponded to depolymerizations of condensed tannins to their monomers which are flavonoids molecules. Under UV at $366 \mathrm{~nm}$, visible yellow spots appeared yellow-green staining.

\section{Structures determination of compounds contained in A, B, C, D extracts}

To determine structures of compounds contained in A, B, C, D extracts, HPLC-ESI ${ }^{+}-$ QTOF-HRMS were used. Superposing chromatographic profiles of the four extracts, we remarked that specific peaks are repeating. All the peaks positions are same but some differ in intensity. Figure 3 (A) displaying extracts obtained at $50{ }^{\circ} \mathrm{C}$, shows the B extract obtained after 3 hours has higher peaks for substances appeared after 14 minutes. Figure 3 (B) displaying extracts obtained at $25{ }^{\circ} \mathrm{C}$, shows almost any difference among peaks of the substances. Figure 3 (C) displaying extracts obtained after 3 hours, shows the B extract obtained at $50^{\circ} \mathrm{C}$ has clearly higher peaks for substances appear after 14 minutes. We then deduced that our proposed extraction methods lead to same chemical compounds extractions but B extract allowed higher concentrations of later substances to be obtained. Identification of those substances was then undertaken, at least in part.

Interesting molecules found belong to terpernoids and flavonoids classes. Identified compounds are: 3-Oxo-12,18-ursadien-28-oic acid belonging to triterpenoids class; Phylloquinol belonging to diterpenoids class; (benzo[d][1,3]dioxol-5-yl)-5,6,7-trimethoxy$4 \mathrm{H}$-chromen-4-one belonging to flavonoids class;3,4-dimethoxyphenyl)-5,6,7,8tetramethoxy-4H-chromen-4-one belonging to flavonoids class; 7,8-tetramethoxy-2-(3,4,5trimethoxyphenyl)-4H-chromen-4-one belonging to flavonoids class. 

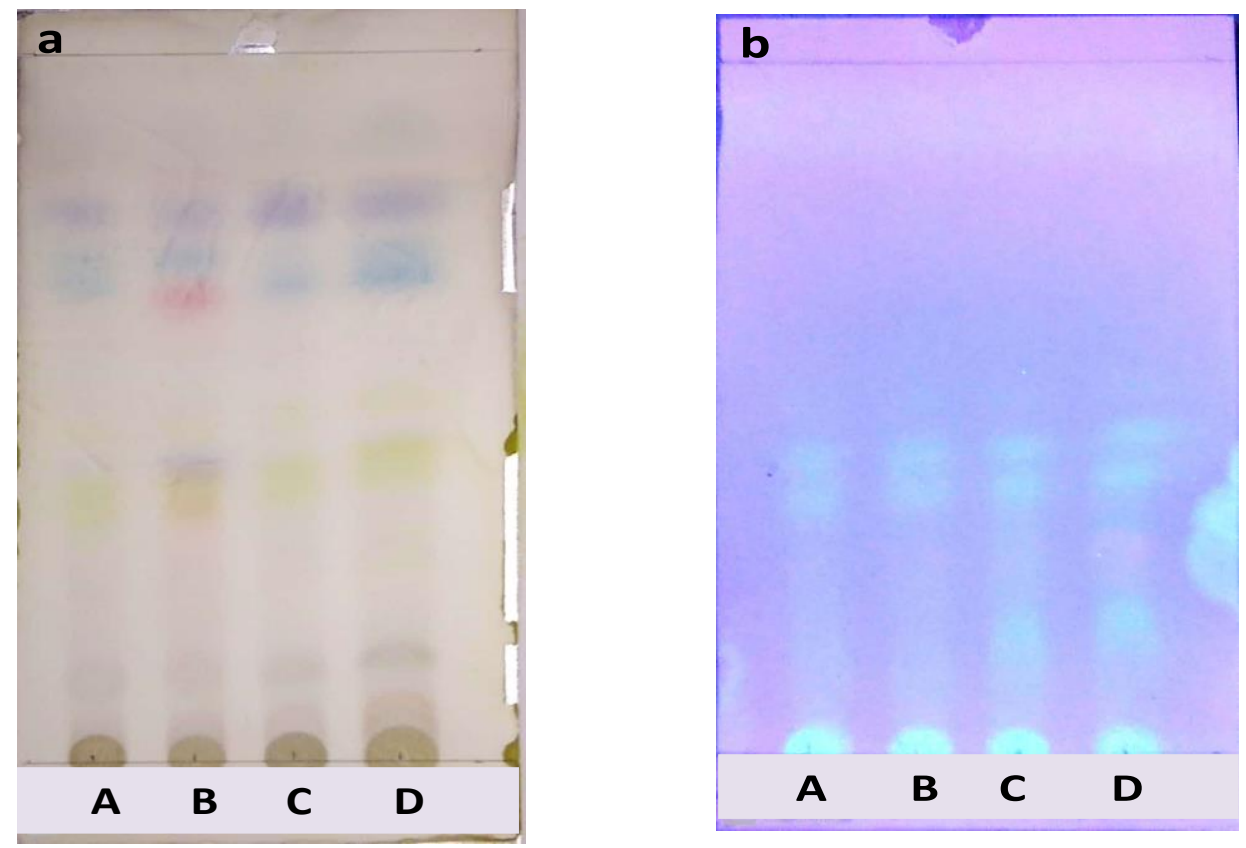

Figure 1: TLC images of the four extracts obtained by A, B, C, D methods resolved with $(30 \%$ Ethyl-acetate; 70\% Dichoromethan). Chromatograms were sprayed with (a) vanillin-sulfuric reagent and (b) chloride aluminium
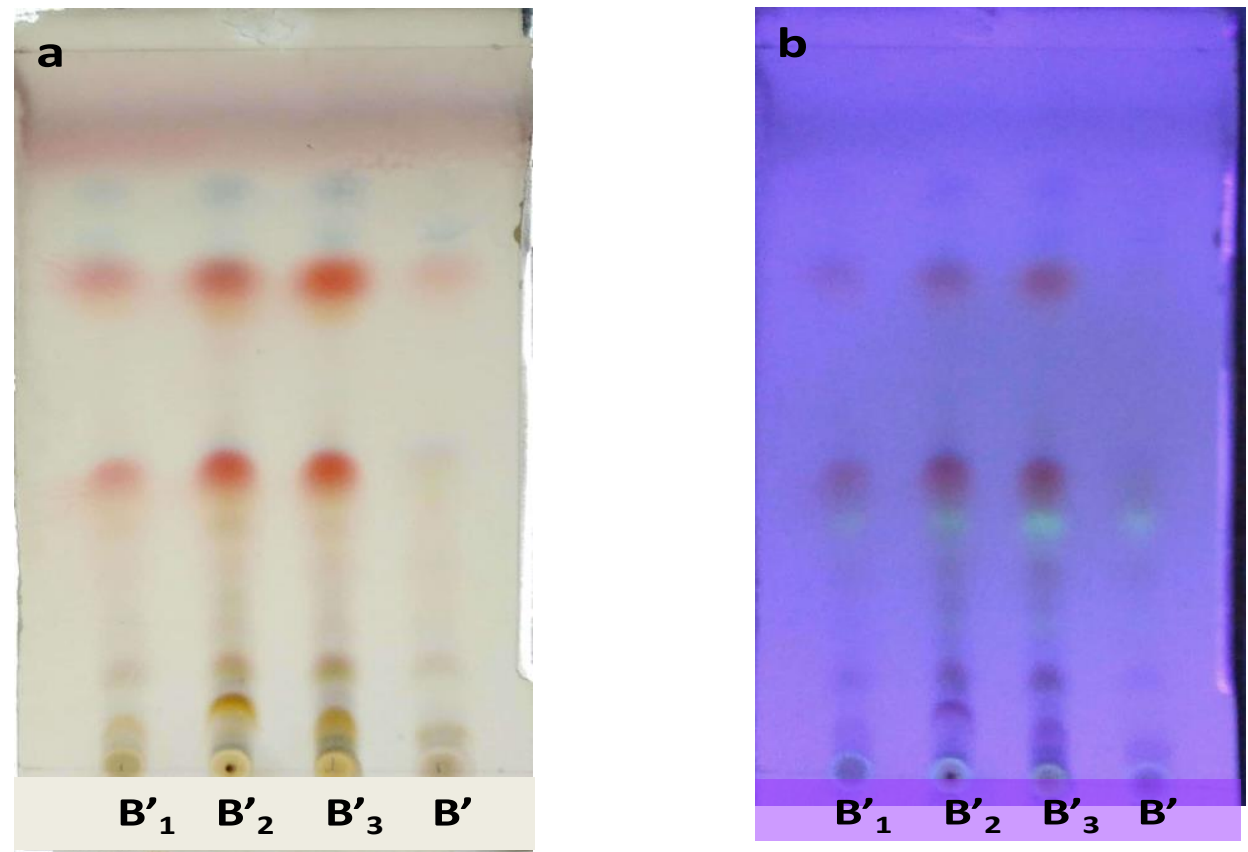

Figure 2: TLC images of $B$ extracts dissolved in sodium saline solution (B') and obtained at one interval week: 10 $0^{\text {th }}\left(B^{\prime} 1\right), 17^{\text {th }}$ (B'2) and 24 $4^{\text {th }}$ August (B'3). B' was obtained in May. Chromatograms were sprayed with (a) vanillin-sulfuric reagent and (b) vanillin-sulfuric reagent + UV (366 nm). 
Table 1: TLC analysis of the four extracts obtained by A, B, C, D methods after Vanillin Sulfuric or Chloride Aluminium pulverizations.

\begin{tabular}{|c|c|c|c|c|c|}
\hline \multirow{3}{*}{ Extracts } & \multicolumn{5}{|c|}{ After Vanillin Sulfuric or Chloride Aluminium pulverization } \\
\hline & \multicolumn{2}{|c|}{$\begin{array}{l}\text { Vanillin Sulfuric } \\
\text { Visible }\end{array}$} & \multicolumn{2}{|c|}{$\begin{array}{l}\text { Chloride Aluminium } \\
\text { + UV } 366 \mathrm{~nm}\end{array}$} & \multirow{2}{*}{$\begin{array}{c}\text { Supposed } \\
\text { molecules }\end{array}$} \\
\hline & Rf & Colour & $\mathrm{Rf}$ & Fluorescence & \\
\hline \multirow{4}{*}{$\begin{array}{l}\text { A: May } 18^{\text {th }} \\
50^{\circ} \mathrm{C}-1 \mathrm{H} \\
\begin{array}{c}\text { Solubilized in } \\
\text { ethanol }\end{array}\end{array}$} & $\begin{array}{l}0,00 \\
0,11\end{array}$ & $\begin{array}{l}\text { Brown } \\
\text { Dark green }\end{array}$ & $\begin{array}{l}0,00 \\
0,23\end{array}$ & $\begin{array}{l}\text { Yellow-green } \\
\text { Yellow-green }\end{array}$ & Polyhydroxyflavones \\
\hline & $\begin{array}{l}0,36 \\
0,45\end{array}$ & $\begin{array}{l}\text { Yellow } \\
\text { Pale yellow }\end{array}$ & $\begin{array}{l}0,36 \\
0,42 \\
0,50 \\
\end{array}$ & $\begin{array}{l}\text { Yellow-green } \\
\text { Yellow-green } \\
\text { Yellow-green }\end{array}$ & $\begin{array}{l}\text { Oligohydroxy / } \\
\text { Oligomethoxyflavones }\end{array}$ \\
\hline & 0,68 & Blue & & & $\begin{array}{l}\text { Triterpene/ Flavanones, flavonols, } \\
\text { methoxyflavones }\end{array}$ \\
\hline & $\begin{array}{l}0,77 \\
0,87\end{array}$ & $\begin{array}{l}\text { Purple } \\
\text { Pale purple }\end{array}$ & 0,90 & Yellow-green & $\begin{array}{l}\text { Triterpene } \\
\text { Stigmasterol }\end{array}$ \\
\hline B: May $24^{\text {th }}$ & $\begin{array}{l}0,00 \\
0,12\end{array}$ & $\begin{array}{l}\text { Brown } \\
\text { Dark green }\end{array}$ & $\begin{array}{l}0,00 \\
0,24 \\
\end{array}$ & $\begin{array}{l}\text { Yellow-green } \\
\text { Yellow-green }\end{array}$ & Polyhydroxyflavones \\
\hline \multirow{3}{*}{$\begin{array}{c}50^{\circ} \mathrm{C}-3 \mathrm{H} \\
\begin{array}{c}\text { Solubilized in } \\
\text { ethanol }\end{array}\end{array}$} & $\begin{array}{l}0,29 \\
0,36 \\
0,47\end{array}$ & $\begin{array}{l}\text { Pale orange } \\
\text { Yellow-orange } \\
\text { Pale yellow }\end{array}$ & $\begin{array}{l}0,37 \\
0,43 \\
0,50\end{array}$ & $\begin{array}{l}\text { Yellow-green } \\
\text { Yellow-green } \\
\text { Yellow-green }\end{array}$ & $\begin{array}{l}\text { Triterpene / Oligohydroxy/ } \\
\text { Oligomethoxyflavones }\end{array}$ \\
\hline & $\begin{array}{l}0,66 \\
0,71\end{array}$ & $\begin{array}{l}\text { Pink } \\
\text { Blue }\end{array}$ & & & $\begin{array}{l}\text { Triterpene/ Flavanones, } \\
\text { Triterpene/ flavonols, methoxyflavones }\end{array}$ \\
\hline & $\begin{array}{l}0,77 \\
0,89 \\
\end{array}$ & $\begin{array}{l}\text { Purple } \\
\text { Pale purple }\end{array}$ & 0,89 & Yellow-green & $\begin{array}{l}\text { Triterpene } \\
\text { Stigmasterol } \\
\end{array}$ \\
\hline \multirow{4}{*}{$\begin{array}{l}\text { C: May } 27^{\text {th }} \\
25^{\circ} \mathrm{C}-1 \mathrm{H} \\
\begin{array}{c}\text { Solubilized in } \\
\text { ethanol }\end{array}\end{array}$} & $\begin{array}{l}0,00 \\
0,13\end{array}$ & $\begin{array}{l}\text { Brown } \\
\text { Dark green }\end{array}$ & $\begin{array}{l}0,00 \\
0,24 \\
\end{array}$ & $\begin{array}{l}\text { Yellow-green } \\
\text { Yellow-green } \\
\end{array}$ & Polyhydroxyflavones \\
\hline & $\begin{array}{l}0,39 \\
0,49 \\
\end{array}$ & $\begin{array}{l}\text { Yellow } \\
\text { Pale yellow }\end{array}$ & $\begin{array}{l}0,28 \\
0,38 \\
0,43 \\
0,50 \\
\end{array}$ & $\begin{array}{l}\text { Yellow-green } \\
\text { Yellow-green } \\
\text { Yellow-green } \\
\text { Yellow-green } \\
\end{array}$ & $\begin{array}{l}\text { Oligohydroxy / } \\
\text { Oligomethoxyflavones }\end{array}$ \\
\hline & 0,64 & Blue & & & $\begin{array}{l}\text { Triterpene/ Flavanones, flavonols, } \\
\text { methoxyflavones }\end{array}$ \\
\hline & $\begin{array}{l}0,78 \\
0,88 \\
\end{array}$ & $\begin{array}{l}\text { Purple } \\
\text { Pale purple }\end{array}$ & 0,89 & Yellow-green & $\begin{array}{l}\text { Triterpene } \\
\text { Stigmasterol }\end{array}$ \\
\hline \multirow{4}{*}{$\begin{array}{l}\text { D: May } 30^{\text {th }} \\
25^{\circ} \mathrm{C}-3 \mathrm{H} \\
\begin{array}{c}\text { Solubilized in } \\
\text { ethanol }\end{array}\end{array}$} & $\begin{array}{l}0,00 \\
0,14\end{array}$ & $\begin{array}{l}\text { Brown } \\
\text { Dark green }\end{array}$ & $\begin{array}{l}0,00 \\
0,24 \\
\end{array}$ & $\begin{array}{l}\text { Yellow-green } \\
\text { Yellow-green }\end{array}$ & Polyhydroxyflavones \\
\hline & $\begin{array}{l}0,41 \\
0,50 \\
\end{array}$ & $\begin{array}{l}\text { Yellow } \\
\text { Pale yellow }\end{array}$ & $\begin{array}{l}0,30 \\
0,39 \\
0,45 \\
0,51 \\
\end{array}$ & $\begin{array}{l}\text { Yellow-green/Pink } \\
\text { Yellow-green } \\
\text { Yellow-green } \\
\text { Yellow-green } \\
\end{array}$ & $\begin{array}{l}\text { Oligohydroxy / } \\
\text { Oligomethoxyflavones }\end{array}$ \\
\hline & 0,69 & Blue & & & $\begin{array}{l}\text { Triterpene/ Flavanones, flavonols, } \\
\text { methoxyflavones }\end{array}$ \\
\hline & $\begin{array}{l}0,79 \\
0,88\end{array}$ & $\begin{array}{l}\text { Purple } \\
\text { Pale purple }\end{array}$ & 0,90 & Yellow-green & $\begin{array}{l}\text { Triterpene } \\
\text { Stigmasterol }\end{array}$ \\
\hline
\end{tabular}

Polyhydroxyflavones $\mathrm{Rf}=0,00-0,25$; oligohydroxy and oligomethoxyflavones have $\mathrm{Rf}=0,3-0,5$; flavanones, flavonols, methoxyflavones $\mathrm{Rf}=0,5-0,75$. 
Table 2: Analysis of stability or evolution of chemical compounds contained in the B type extracts solubilized in sodium saline solution.

\begin{tabular}{|c|c|c|c|c|c|}
\hline \multirow[t]{3}{*}{ Extracts } & \multicolumn{5}{|c|}{ After Vanillin Sulfuric pulverization } \\
\hline & \multicolumn{2}{|c|}{$\begin{array}{l}\text { Vanillin Sulfuric } \\
\text { Visible }\end{array}$} & \multicolumn{2}{|c|}{$\begin{array}{l}\text { Vanillin Sulfuric } \\
+ \text { UV } 366 \mathrm{~nm}\end{array}$} & \multirow{2}{*}{$\begin{array}{l}\text { Supposed } \\
\text { molecules }\end{array}$} \\
\hline & $\mathrm{Rf}$ & Colour & $\mathrm{Rf}$ & Fluorescence & \\
\hline \multirow[t]{2}{*}{$\mathrm{B}_{1}{ }_{1}$ : August $10^{\text {th }}$} & 0,00 & Brown-orange & 0,00 & Blue & Triterpene/ Polyhydroxyflavone \\
\hline & $\begin{array}{l}0,06 \\
0,12\end{array}$ & $\begin{array}{l}\text { Pale orange } \\
\text { Orange-brown }\end{array}$ & & & $\begin{array}{l}\text { Triterpene/ Polyhydroxyflavone } \\
\text { Triterpene/ Polvhydroxvflavone }\end{array}$ \\
\hline \multirow[t]{3}{*}{$\begin{array}{l}\text { Solubilized in } \\
\text { sodium saline } \\
\text { solution }\end{array}$} & 0,40 & Pink & $\begin{array}{l}0,35 \\
0,41\end{array}$ & $\begin{array}{l}\text { Yellow-green } \\
\text { Pink }\end{array}$ & $\begin{array}{l}\text { Triterpene / Oligohydroxy / } \\
\text { Oligomethoxyflavones }\end{array}$ \\
\hline & 0,68 & Pink & & & $\begin{array}{l}\text { Triterpene / Flavanones, flavonols, } \\
\text { methoxyflavones }\end{array}$ \\
\hline & $\begin{array}{l}0,80 \\
0,87\end{array}$ & $\begin{array}{l}\text { Blue } \\
\text { Purple }\end{array}$ & & & $\begin{array}{l}\text { Triterpene } \\
\text { Stigmasterol }\end{array}$ \\
\hline \multirow{6}{*}{$\begin{array}{l}\mathrm{B}_{2}{ }_{2} \text { : August } 17^{\text {th }} \\
50^{\circ} \mathrm{C}-3 \mathrm{H} \\
\text { Solubilized in } \\
\text { sodium saline } \\
\text { solution }\end{array}$} & 0,00 & Orange & 0,00 & Blue & Triterpene/ Polyhydroxyflavone \\
\hline & 0,08 & Dark orange & 0,09 & Purple & Triterpene/ Polyhydroxyflavone \\
\hline & 0,15 & Brown-orange & 0,14 & Purple & Triterpene/ Polyhydroxyflavone \\
\hline & 0,41 & Red & $\begin{array}{l}0,35 \\
0,42\end{array}$ & $\begin{array}{l}\text { Yellow-green } \\
\text { Pink }\end{array}$ & $\begin{array}{l}\text { Tannin/Anthocyanidols/ } \\
\text { Oligohydroxy/ Oligomethoxyflavones }\end{array}$ \\
\hline & 0,68 & Pink-orange & 0,68 & Pink-purple & $\begin{array}{lll}\begin{array}{l}\text { Triterpene / } \\
\text { methoxyflavones }\end{array} & \text { Flavanones, } & \text { flavonols, } \\
\end{array}$ \\
\hline & $\begin{array}{l}0,80 \\
0,89\end{array}$ & $\begin{array}{l}\text { Blue } \\
\text { Purple }\end{array}$ & & & $\begin{array}{l}\text { Triterpene } \\
\text { Stigmasterol }\end{array}$ \\
\hline \multirow[t]{3}{*}{$\mathrm{B}_{3}{ }_{3}$ : August $24^{\text {th }}$} & $\begin{array}{l}0,00 \\
0,04\end{array}$ & $\begin{array}{l}\text { Orange } \\
\text { Green-brown }\end{array}$ & 0,00 & & Triterpene/ Polyhydroxyflavones \\
\hline & 0,07 & Orange & 0,08 & Purple & Triterpene/ Polyhydroxyflavones \\
\hline & 0,14 & Brown-orange & 0,14 & Purple & Triterpene/ Polyhydroxyflavones \\
\hline \multirow[t]{4}{*}{$\begin{array}{l}\text { Solubilized in } \\
\text { sodium saline } \\
\text { solution }\end{array}$} & 0,41 & Red & $\begin{array}{l}0,35 \\
0,42\end{array}$ & $\begin{array}{l}\text { Yellow-green } \\
\text { Pink }\end{array}$ & $\begin{array}{l}\text { Tannin/Anthocyanidols/ } \\
\text { Oligohvdrox v/ Oligomethoxyflavones }\end{array}$ \\
\hline & $\begin{array}{l}0,68 \\
0,75\end{array}$ & $\begin{array}{l}\text { Orange-red } \\
\text { Blue }\end{array}$ & 0,68 & Pink & $\begin{array}{l}\text { Triterpene/ Flavanones, flavonols, } \\
\text { Triterpene/ methoxyflavones }\end{array}$ \\
\hline & 0,81 & & & & \\
\hline & 0,89 & Purple & & & Stigmasterol \\
\hline \multirow{2}{*}{ 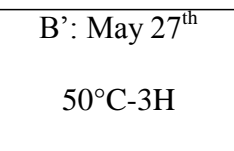 } & $\begin{array}{l}0,00 \\
0,04\end{array}$ & $\begin{array}{l}\text { Brown } \\
\text { Pale Dark-green }\end{array}$ & 0,00 & $\begin{array}{l}\text { Blue } \\
\text { Purnle }\end{array}$ & Triterpene/ Polyhydroxyflavones \\
\hline & 0,14 & Pale Brown & & & \\
\hline \multirow{3}{*}{$\begin{array}{l}\text { Solubilized in } \\
\text { sodium saline } \\
\text { solution }\end{array}$} & & & 0,35 & Yellow-green & Oligohydroxy / Oligomethoxyflavones \\
\hline & $\begin{array}{l}0,68 \\
0,76\end{array}$ & $\begin{array}{l}\text { Pink } \\
\text { Blue }\end{array}$ & & & $\begin{array}{lll}\text { Triterpene } & \\
\text { Triterpene/ } & \text { Flavanones, flavonols, } \\
\text { methoxyflavones } & \end{array}$ \\
\hline & 0,89 & Purple & & & Stigmasterol \\
\hline
\end{tabular}

Polyhydroxyflavones $R f=0,00-0,25$; oligohydroxy and oligomethoxyflavones have $R f=0,3-0,5$; flavanones, flavonols, methoxyflavones $R f=0,5-0,75$. 
Table 3: Intensity analysis of chemical compounds contained in the B type extracts solubilized in sodium saline solution. (A) in visible after Vanilin Sulfuric pulverization and (B) fluorescence at $366 \mathrm{~nm}$ UV light after Vanilin Sulfuric pulverization.

(A)

\begin{tabular}{|c|c|c|c|c|}
\hline Rf & B': May $27^{\text {th }}$ & B'1: August 10 $^{\text {th }}$ & B'2: August 17 $7^{\text {th }}$ & B'3: August 24 ${ }^{\text {th }}$ \\
\hline 0.00 & Brown (+) & $\begin{array}{c}\text { Brown-Orange } \\
(++)\end{array}$ & Orange $(+++)$ & Orange $(++)$ \\
\hline 0.04 & $\begin{array}{c}\text { Pale dark Green } \\
\qquad(+)\end{array}$ & - & - & $\begin{array}{c}\text { Brown-Green } \\
(+++)\end{array}$ \\
\hline $0.06 / 0.07 / 0.08$ & - & Pale Orange $(+)$ & Dark Orange $(++)$ & Orange $(++)$ \\
\hline $0.12 / 0.14 / 0.15$ & Pale Brown (+) & Brown-Orange $(+)$ & $\begin{array}{c}\text { Brown-Orange } \\
(++)\end{array}$ & $\begin{array}{c}\text { Brown-Orange } \\
(++)\end{array}$ \\
\hline 0.40 & - & Pink (++) & $\operatorname{Red}(+++)$ & $\operatorname{Red}(+++)$ \\
\hline 0.68 & Pink (+) & Pink (++) & $\begin{array}{c}\text { Pink-Orange } \\
(+++)\end{array}$ & Orange-Red $(+++)$ \\
\hline 0.76 & Blue (+) & - & - & - \\
\hline $0.80 / 0.81$ & - & Blue (+) & Blue $(++)$ & Blue $(++)$ \\
\hline $0.87 / 0.89$ & Purple (+) & Purple (+) & Purple (+) & Purple (+) \\
\hline
\end{tabular}

$(+++)$ : high intensity; $(++)$ : weak intensity; (+): trace; (-): absence.

(B)

\begin{tabular}{ccccc}
\hline Rf & B': May 27 $^{\text {th }}$ & B'1: August 10 $^{\text {th }}$ & B'2: August 17 $^{\text {th }}$ & B'3: August 24 $^{\text {th }}$ \\
\hline 0.00 & Blue (+) & Blue (+) & Blue (+) & Blue (+) \\
0.05 & Purple (+) & - & - & - \\
$0.08 / 0.09$ & - & - & Purple (++) & Purple (++) \\
0.14 & - & - & Purple (++) & Purple (++) \\
0.35 & Yellow-Green (+) & Yellow-Green (+) & Yellow-Green (+) & $\begin{array}{c}\text { Yellow-Green } \\
(++)\end{array}$ \\
& & & & \\
$0.41 / 0.42$ & - & Pink (++) & Pink (+++) & Pink (+++) \\
0.68 & - & Pink-Purple (++) & Pink (++) \\
\hline
\end{tabular}



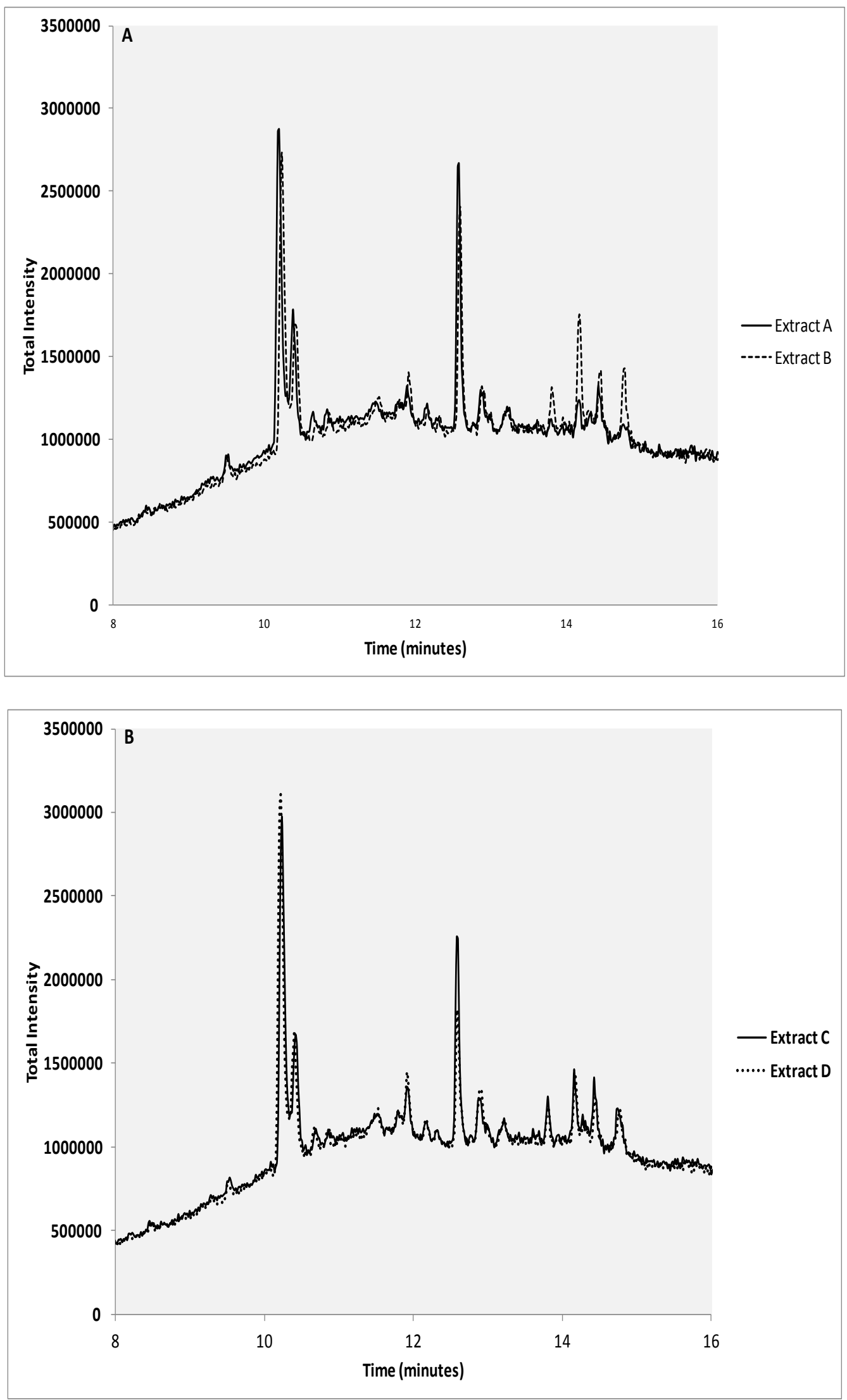


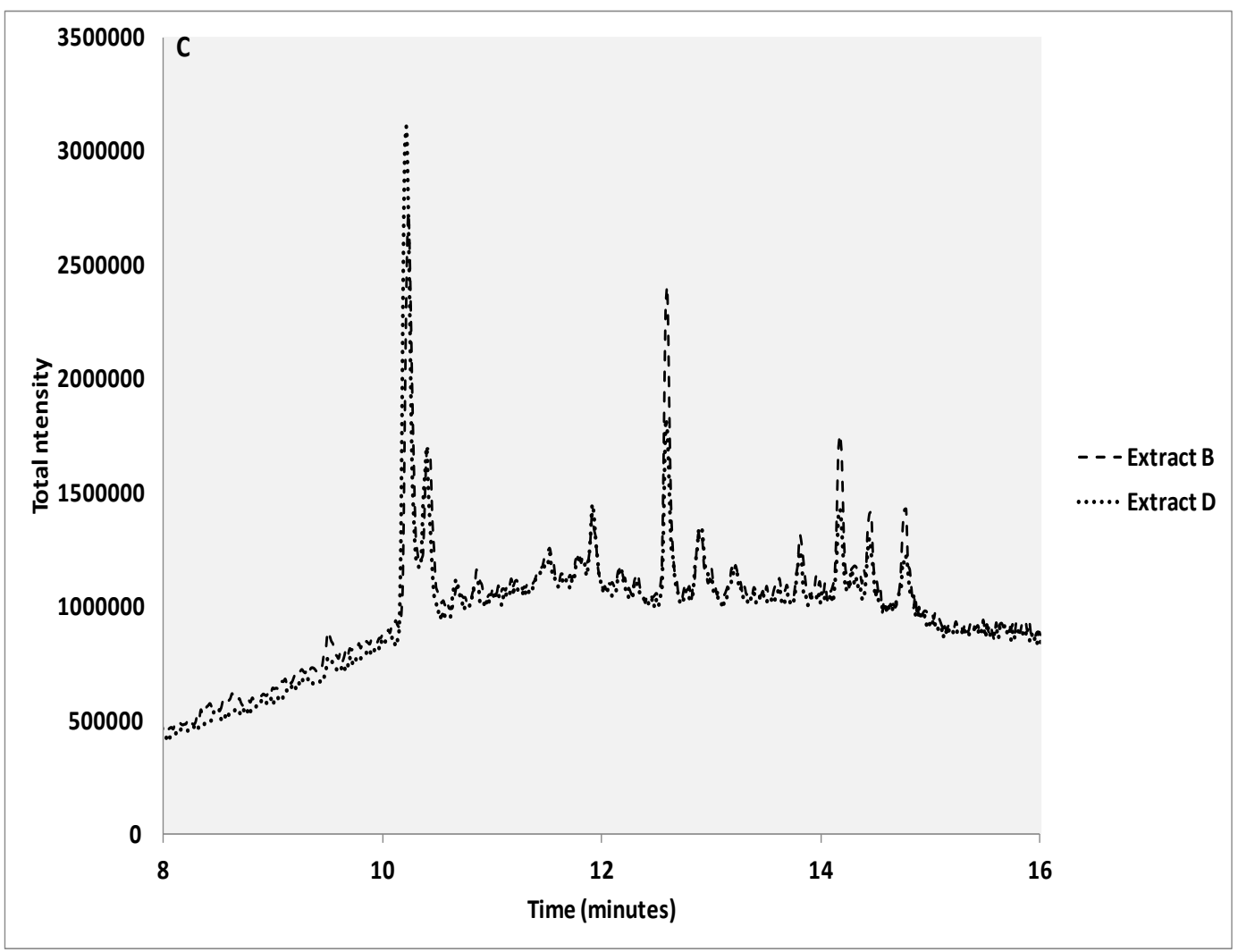

Figure 3: Comparative chromatographic profiles of the different extracts. (A): extracts $\mathrm{A}$ and $\mathrm{B}$ obtained at $50{ }^{\circ} \mathrm{C} ;(\mathbf{B})$ : extracts $\mathrm{C}$ and D obtained at $25^{\circ} \mathrm{C} ;(\mathbf{C})$ : extracts $\mathrm{B}$ and D obtained after 3 hours.

\section{DISCUSSION}

Since dawn of time plants are used as rich source of medicine. Ageratum conyzoides Linn. is widely utilized in traditional medicine systems wherever it grows, although applications vary by region: in Asia, South America and Africa, aqueous extract of this plant is used as a bactericide (Tailor and Goyal, 2012).

Our TLC results, presented several types of flavonoids in all extracts studied, particularly in $\mathrm{B}$ one $\left(50{ }^{\circ} \mathrm{C}-3 \mathrm{H}\right)$. This richness in flavonoids forced their identification.

Flavonoids are composed of two phenyls cycles $\mathrm{C} 6$, cycles $\mathrm{A}$ and $\mathrm{B}$, connected by a three carbons bridge (C6-C3-C6 structure). The last one is situated between cycle $\mathrm{A}$ and cycle $\mathrm{B}$ and is commonly cyclized to form the central $\mathrm{C}$ cycle. Flavonoids can be subdivided into different subgroups depending on the carbon of the $\mathrm{C}$ ring on which the $\mathrm{B}$ ring is attached and the degree of unsaturation and oxidation of the $\mathrm{C}$ ring (Panche et al., 2016). Flavonoids in which the $\mathrm{B}$ ring is linked in position 3 of the $\mathrm{C}$ ring are called isoflavones. Those in which the $\mathrm{B}$ ring is linked in position 4 are called neoflavonoids, while those in which the $\mathrm{B}$ ring is linked in position 2 can be further subdivided into several subgroups on the basis of the structural features of the $\mathrm{C}$ ring (Panche et al., 2016). These subgroups are: flavones, flavonols, flavanones, flavanols or cathechins, anthocyanins and chalcones (Panche et al., 2016). The wide variety of flavonoids in nature and their broad biological activity is due to the many classes of substituting groups (i.e.: hydroxyl-, methyl-, acetyl- groups) (Mozzicafreddo et al., 2006). The fusion of A and $\mathrm{C}$ cycles leads to the formation a chromene (or benzopyrane) ring.

Understanding the structural chemistry of condensed tannins and flavonoids is 
essential to proper use of the vanillin assay (Hagerman, 2002). Condensed tannins or proanthocyanidols are polyphenols belong to the flavonoids family. They are chemically defined as oligomers or flavonoids units polymers, the monomer being a flavan-3-ol: cathechin. The vanillin reaction involves the meta-substituted ring of flavanols to yield a red adduct. Although the vanillin reaction has been widely used to estimate condensed tannin (proanthocyanidin), the reaction is not specific for condensed tannins. Any appropriately substituted flavanol reacts in the assay (Hagerman, 2002). Then, vanillin reacts only with free flavan-3-ols or with the terminal unit of the proanthocyanidins (Hagerman, 2002). To date, TLC detection of catechins has mainly been performed with the aromatic aldehyde vanillin (Glavnik et al., 2009). The attenuation of red color with time of our B extracts could then be understood by an eventual depolymerisation of proanthocyanidins in its monomer (catechins). According to Lhuillier (2007), that substances which appeared in red with vanillin sulfuric acid are tannins.

Flavan-3-ols (catechins) are polyphenolic compounds that have been shown to exhibit a wide variety of beneficial effects on human health (Vovk et al., 2005).

Triterpenes are molecules with 30 atoms of Carbon. Most of them are under esterified free form or heterosidic free form. Steroids are not terpenes but biodegradation products of triterpenes. They are composed of four rings as all sterols. However, for many biochemical, sterols are an entire familly which includes steroids. Analyzed samples seemed contain triterpenes (purple, pink and orange colors reveal with vanillin sulfuric acid) and sterols (blue color). As suggested by Lhuillier (2007) blue-purple coloration corresponded to triterpenes. We proceeded to revelation with polyvalent vanillin sulfuric acid to allow first orientation for different classes of composed substances present in samples.

Distance of migration or, more absolutely Rf values of substances extracted, depend essentially on their polarity: polyhydroxyflavones have low Rf values $(0,00-0,25) ; \quad$ oligohydroxy and oligomethoxyflavones have $\mathrm{Rf}$ values comprise between (0,3-0,5). Flavanones, flavonols, methoxyflavones have more elevated $\mathrm{Rf}$ values comprise between $(0,5$ $0,75)$. The TLC of our extracts presented substances belong to each category of this classification.

Chloride aluminum $\left(\mathrm{AlCl}_{3}\right)$ showed the presence of several different spots of fluorescence, which revealed the flavonoids in yellow in the visible and under UV/366 nm or fluorescent yellow-green (Konan et al., 2012) (Table 1).

Phytochemicals have exhibited potent activities while many researchers have used natural products to act against bacterial resistance. These agents can act alone or in combination with antibiotics to enhance the antibacterial activity against a wide range of bacteria (Khameneh et al., 2019). There is high variability in the secondary metabolites of Ageratum conyzoides Linn. which include flavonoids, alkaloids, cumarins, essential oils and tannins (Tailor and Goyal, 2012). The second and the fourth steps of our extraction methods displayed nauseating and good smelling odor respectively and residues obtained were around $80 \%$ soluble in aqueous sodium saline solution whereas they were completely soluble in ethanol. We then deduced that our extracts contain a certain amount of oil. According to Singh et al. (2013) two corroborated facts are presented: first, the essential oil obtained by steam distillation has been reported to have a powerful nauseating odor and second, the essential oil of Ageratum conyzoides Linn. has very strong smell.

The most common component of the essential oil of Ageratum conyzoides Linn. is 7-methoxy2,2-dimethylchromene (precocene1) (Tailor and Goyal, 2012). Whereas this substance was not yet identified in our extracts, genus Ageratum is well known for its chromenes and flavonoids contents (Singh et al., 2013) and we found those compounds in our extracts.

In fact, Ageratum conyzoides Linn. is rich in polyoxygenated flavonoids among which 14 polymethoxylated flavones. These polyhydroxyflavones include quercetin, kaempferol and their glycosides, too (Tailor 
and Goyal, 2012). They are namely scutellarein-5,6,7,1-tetrahydroxyflavone, quercetin-3-rhamnopiranoside, kaempferol 3,7-diglucopiranoside (Singh et al., 2013). Surprisingly, any trace of quercetin was found in our extracts but 3 chromenes: to date, 2(benzo[d][1,3]dioxol-5-yl)-5,6,7-trimethoxy4H-chromen-4-one; 2-(3,4-dimethoxyphenyl)5,6,7,8-tetramethoxy-4H-chromen-4-one;

5,6,7,8-tetramethoxy-2-(3,4,5-

trimethoxyphenyl)-4H-chromen-4-one. These substances were yet identify as structures 33 , 24 and 23 respectively in Favonoids class (Tailor and Goyal, 2012).

Ageratum conyzoides Linn. contains the two major common sterols, sitosterol and stigmasterol and the oil from leaves and stems of this plant was reported to contain sterols like friedeline, beta-sitosterol and stigmasterol as major constituents and minor sterol include brassicasterol (Singh et al., 2013).

The presence of beta-sitosterol and stigmasterol in various tissue samples and plants parts of Ageratum conyzoides Linn. were confirmed by thin layer chromatography (TLC) for beta-sitosterol ( $\mathrm{Rf}=0,95)$ and stigmasterol $(\mathrm{Rf}=0,89)$ (Singh et al., 2013) and we retrieve this substance in our TLC analysis.

Phenolic compounds showed diverse mechanisms of action against different bacterial strains from synergistic activity via inhibition of efflux pumps (EPs), interacting with the cell membrane and inhibition of cell wall biosynthesis to inhibition of certain critical enzymes including urease, sortase A and dihydrofolate reductase. The observed activities were remarkable in some studies making phenolic compounds a good candidate for future in vivo studies and even clinical trials.

Terpenes are the major ingredients of herbal resins and responsible for the common fragrance of various plants. We found in our extracts 3-Oxo-12,18-ursadien-28-oic acid, a triterpenoid, and Phylloquinol, a diterpenoid. Commonly, Gram-positive bacteria are more susceptible to terpenes than Gram-negative ones. The antimicrobial mechanism of terpenes is closely associated with their lipophilic features. Monoterpenes preferentially impact on the structures of the membrane through increasing its fluidity and permeability, altering the topology of its proteins and making disturbances across the respiration chain (Khameneh et al., 2019).

A number of studies have been conducted to elucidate the structure-activity relationships (SARs) of flavonoids for their biological activities. According to Liu et al. (2010), eleven flavonoids were shown to produce dose-dependently inhibitory activities on thrombin. They demonstrated that a hydroxyl group at C-3 played a key role in the inhibitory activity and more $\mathrm{OH}$ groups in the B-ring could increase thrombin inhibition activity. Furthermore, it was found that the presence of $\mathrm{C}(2)=\mathrm{C}(3)$ and $\mathrm{C}(4)=\mathrm{O}$ bonds were very important in showing the activity (Liu et al., 2010). Though we know our extracts inhibit thrombin activity (by elongating gel time), we have not yet clearly identified those structures. Triterpenoids from ginseng, ginsenosides, have also been reported to possess anti-coagulation properties in vitro (Xiong et al., 2017). These authors reported many other studies demonstrating anticoagulation effect of triterpenoids named ginsenosides.

\section{Conclusion}

Our main objective starting this work was to proposed four (4) methods of soft aqueous extraction of leaves of Ageratum conyzoides Linn., a well known west African plant as it was yet known for its wound healing properties. However, we specifically searched tannins and flavonoids and our extracts contain several types of flavonoids at monomer and polymeric forms. The TLC chromatograms of the B extract obtained at 50 ${ }^{\circ} \mathrm{C}$ after 3 hours showed more spots and the same extract HPLC-ESI+-QTOF-HRMS chromatogram showed higher peaks intensity.

At the end of this work, we have not completely identified all chemical compounds structures, which would allow us to understand how those extracts could reversibly or irreversibly inhibit serine proteases like thrombin or those present in bacterial. This will be complete in a next work. 


\section{COMPETING INTERESTS}

The authors have not declared any conflict of interests.

\section{AUTHORS CONTRIBUTIONS}

AEA conceived the research idea, made the extractions, analyzed the data and wrote the draft. IKS and KE provided technical and experimental assistance for TLC and HPLC-ESI ${ }^{+}$-QTOF analysis and they made valuable comments on the manuscript. KK gave valuable scientific critical advises.

\section{ACKNOWLEDGEMENTS}

We would also like to extend our gratitude to Professor TETE-BENISSAN A.K.C, Department of Biochemistry, University of Lome and to the anonymous reviewers for their valuable comments and suggestions made to help improve the manuscript.

\section{REFERENCES}

Akpalo E, Tété-Bénissan A, Awaga KA, Akpagana K. 2015. Review of twelve West Africa medicinal plants: active phytochemical combinations in direct biochemically wound healing process. Journal of Medicinal Plants Research, 9: 908-917.

DOI: https://doi.org/10.5897/JMPR2015.5849

Cieśla $Ł$, Staszek D, Hajnos M, Kowalska T, Waksmundzka-Hajnos M. 2011. Development of chromatographic and free radical scavenging activity fingerprints by thin-layer chromatography for selected Salvia species. Phytochemical Analysis, 22: 5965.

DOI: https://doi.org/10.1002/pca.1251

Glavnik V, Simonovska B, Vovk I. 2009. Densitometric determination of (+)catechin and (-)-epicatechin by 4dimethylaminocinnamaldehyde reagent. Journal of Chromatography, 1216: 4485-4491.

DOI: https://doi.org/10.1016/j.chroma.2009.03 .026

Hagerman AE. 2002. Tannin chemistry. http://www.users.muohio.edu/hagermae

Kalan L, Grice EA. 2018. Fungi in the Wound Microbiome. Advances in Wound
Care, 7:

247-255.

DOI: https://doi.org/10.1089/wound.2017.075 6

Khameneh B, Iranshahy M, Soheili V, Fazly Bazzaz BS. 2019. Review on plant antimicrobials: a mechanistic viewpoint. Antimicrobial Resistance \& Infection Control, 8.8 DOI: https://doi.org/10.1186/s13756-0190559-6

Lhuillier A. 2007. Contribution à l'étude phytochimique de quatre plantes malgaches: Agauria salicifolia Hook. $\mathrm{F}$ Ex Olivier, Agauria polyphylla Baker (Ericaceae), Tambourissa trichophylla Baker (Monimiaceae) et Embelia concinna Baker (Myrsinaceae). PhD thesis, Institut National Polytechnique de Toulouse, Toulouse, $\mathrm{N}^{\circ}$ d'ordre : 2467.

Li K, Diao Y, Zhang H, Wang S, Zhang Z, Yu B, Huang S, Yang H. 2011. Tannin extracts from immature fruits of Terminalia chebula Fructus Retz. promote cutaneous wound healing in rats. BMC Complementary and Alternative Medicine, 11. DOI: https://doi.org/10.1186/1472-6882-11-86

Liu L, Ma H, Yang N, Tang Y, Guo J, Tao W, Duan J. 2010. A Series of Natural Flavonoids as Thrombin Inhibitors: Structure-activity relationships. Thrombosis Research, 126: e365e378.DOI:

https://doi.org/10.1016/j.thromres.2010.0 8.006

Konan KM, Mamyrbékova-Békro JA, Békro YA, Marcel KK, Akhanovna MBJ. 2012. Quantification of total phenols and flavonoids of Desmodium adscendens (Sw.) DC. (Papillionaceae) and projection of their antioxidant capacity. Journal of Applied Biosciences, 4: 33553362.

Mozzicafreddo M, Cuccioloni M, Eleuteri AM, Fioretti E, Angeletti M. 2006. Flavonoids inhibit the amidolytic activity of human thrombin. Biochimie, 88: 1297-1306.

DOI: https://doi.org/10.1016/j.biochi.2006.04. 007

Panche AN, Diwan AD, Chandra SR. 2016. Flavonoids: an overview. Journal of 
Nutritional Science, 5. DOI: https://doi.org/10.1017/jns.2016.41

Saloufou KI, Boyode PB, Simalou O, Eloh K, Melila M, Kpegba K, Novidzro KM, Gaslonde T, Michel S. 2017. Identification de deux phytostérols biologiquement actifs de l'extrait cyclohexanique des feuilles de Ficus sur (Moraceae). International Journal of Biological and Chemical Sciences, 11: 2510.

DOI:

https://doi.org/10.4314/ijbcs.v11i5.44

Saloufou KI, Boyode P, Simalou O, Eloh K, Idoh K, Melila M, Toundou O, Kpegba K, Agbonon A. 2018. Chemical composition and antioxidant activities of different parts of Ficus sur. J. Herbmed. Pharmacol., 7(3): $\quad$ 185-192. DOI: 10.15171/jhp.2018.30

Singh SB, Devi WR, Devi WI, Swapana N, Singh CB. 2013. Ethnobotany, phytochemistry and pharmacology of Ageratum conyzoides Linn (Asteraceae). Journal of Medicinal Plants Research,
7(8):

DOI:10.5897/JMPR012.897

371-385.

Su X, Liu X, Wang S, Li B, Pan T, Liu D. 2016. Wound-healing promoting effect of total tannins from Entada phaseoloides (L.) Merr. in rats. Burns, 10: 010. DOI: 10.1016/j.burns.2016

Tailor CS, Goyal A. 2012. A Comprehensive Review on Ageratum conyzoides Linn. (Goat weed). Int. J. Pharm. Phytopharmacol. Res., 1(6): 391-395.

Vovk I, Simonovska B, Vuorela H. 2005. Separation of eight selected flavan-3-ols on cellulose thin-layer chromatographic plates. Journal of Chromatography, 1077: 188-194. DOI: https://doi.org/10.1016/j.chroma.2005.03 .096

Xiong L, Qi Z, Zheng B, Li Z, Wang F, Liu J, $\mathrm{Li}$ P. 2017. Inhibitory Effect of Triterpenoids from Panax ginseng on Coagulation Factor X. Molecules, 22: 649.

DOI: https://doi.org/10.3390/molecules220406 49 